\title{
Monoamine Oxidase A in Antisocial Personality Disorder and Borderline Personality Disorder
}

\author{
Nathan J. Kolla ${ }^{1,2,3} \cdot$ Sarah A. Vinette ${ }^{1}$
}

Published online: 3 February 2017

(C) Springer International Publishing AG 2017

\begin{abstract}
Purpose of Review Variation in the monoamine oxidase A (MAO-A) gene and MAO-A enzyme levels have been linked to antisocial behavior and aggression in clinical and non-clinical populations. Here, we provide an overview of the genetic, epigenetic, and neuroimaging research that has examined MAO-A structure and function in antisocial personality disorder (ASPD) and borderline personality disorder (BPD).

Recent Findings The low-activity MAO-A variable nucleotide tandem repeat genetic polymorphism has shown a robust association with large samples of violent and seriously violent offenders, many of whom had ASPD. A recent positron emission tomography (PET) study of ASPD similarly revealed low MAOA density in brain regions thought to contribute to the psychopathology of the condition. By contrast, PET has also demonstrated that brain MAO-A levels are increased in BPD and that they relate to symptoms of low mood and suicidality.

Summary Candidate gene studies have produced the most compelling evidence connecting MAO-A genetic variants to both ASPD and BPD. Still, conflicting results abound in the literature, making it highly unlikely that ASPD or BPD is related to a
\end{abstract}

This article is part of the Topical Collection on Personality and Impulse Control Disorders

Nathan J. Kolla

nathan.kolla@mail.utoronto.ca

1 Centre for Addiction and Mental Health (CAMH), Toronto, ON, Canada

2 Violence Prevention Neurobiological Research Unit, CAMH, Toronto, ON, Canada

3 Department of Psychiatry, University of Toronto, Toronto, ON, Canada specific MAO-A genetic variant. Future research should strive to examine how MAO-A genotypes interact with broadspectrum environmental influences to produce brain endophenotypes that may ultimately become tractable targets for novel treatment strategies.

Keywords Monoamine oxidase A · Antisocial personality disorder · Borderline personality disorder · Psychopathy · Genetics $\cdot$ Neuroimaging

\section{Introduction}

The role of monoamine oxidase A (MAO-A), a mitochondrial enzyme encoded by the X-linked MAO-A gene, in molding aspects of human personality and increasing risk for adverse behaviors has attracted the attention of researchers for over a decade. Although associations have been described between several psychiatric illnesses and altered MAO-A function, antisocial personality disorder (ASPD) and borderline personality disorder (BPD) are especially implicated given that their symptoms of impulsivity and aggression have been the focus of numerous MAO-A candidate gene studies in non-clinical samples. This paper has four goals. The first is to provide a brief overview of the structure and function of MAO-A, concentrating on seminal studies that first described a connection between low or absent enzyme levels and aggression. The second aim describes linkages between in vivo and in vitro markers of MAO-A in ASPD. The third objective will summarize similar evidence pertaining to BPD. Fourth, we conclude with a summary of the findings and offer opportunities for future directions. For all objectives, the most recent evidence in the literature is prioritized. 


\section{MAO-A}

The MAO-A gene includes a 30 bp variable nucleotide tandem repeat (VNTR) polymorphism that is situated $1.2 \mathrm{~kb}$ upstream of the transcription start site in the human promoter [1]. Multiple copies of the MAO-A VNTR exist: 2, 3, 3.5, 4, 5, or 6 [2]. Gene transfusion and transfection assays have demonstrated that the number of copies influences promoter activity and basal transcription rate in an allele-specific manner. For instance, alleles containing 3.5 or 4 copies are transcribed 2-10 times more efficiently than the 2- or 3-repeat VNTR alleles [1, 3]. 3.5- and 4repeat alleles have, therefore, become known as high-activity MAO-A alleles (MAOA-H), whereas 2- and 3-repeat alleles have been designated low-activity MAO-A genetic variants (MAOA-L). MAO-A enzyme in the brain catabolizes serotonin, norepinephrine, and dopamine [4]. It is notable that the results of two positron emission tomography (PET) investigations - one measuring brain MAO-A levels using the radiotracer $\left[{ }^{11} \mathrm{C}\right]$ clorgyline and the other applying $\left[{ }^{18} \mathrm{~F}\right]-\mathrm{FDG}$ PET to assess regional brain metabolism - showed no correspondence with the MAO-A VNTR genotype in a sample of healthy males $[5,6]$. These results suggest that MAO-A may exert its most important effects early in development.

Support for this position comes from animal studies, which have demonstrated that MAO-A knockout mice exhibit more aggressive behavior than wild type [7]. In humans, lack of MAO-A as a result of a nonsense mutation, which has become known as Brunner syndrome, results in a phenotype marked by impulsive, explosive aggression in addition to other cognitive deficits $[8,9]$. However, since in the past 20 years, only two other families have been identified as having members afflicted with Brunner syndrome [10], it is unlikely that congenital deficiency of MAO-A plays a significant role in the genesis of human violence and aggression.

A pivotal investigation explored the interaction of MAO-A genetic variants and environmental influences on a range of criminogenic variables in a population sample [11]. Using longitudinal data from the Dunedin Multidisciplinary Health and Development Study, the authors demonstrated that the relationship between childhood maltreatment and ASPD symptoms among other measures of violence were all moderated by the MAO-A genotype. Specifically, the association of childhood maltreatment with each outcome measure was increased among males with MAOA-L relative to MAOA-H. This study was a catalyst for subsequent research that aimed to investigate MAOA in non-clinical and clinical populations, including ASPD and BPD.

\section{Antisocial Personality Disorder}

ASPD is chronic psychiatric illness that first onsets during youth as conduct disorder. It is a common condition with a prevalence of $7 \%$ in the community [12] and 50\% among incarcerated individuals [13]. Individuals with ASPD frequently act impulsively and engage in repeated bouts of aggressive behavior [14]. The neural underpinnings of ASPD are poorly understood and robust treatments are lacking, hence the need to accelerate research in this area. Genetic, epigenetic, and neuroimaging studies have all investigated MAO-A in relation to ASPD.

\section{MAO-A VNTR in ASPD}

It is important to note that in contrast to the landmark Dunedin study described above, both main and interactive effects of the MAO-A VNTR have been associated with ASPD and its symptomatology. An early investigation first reported an increased frequency of MAOA-L in ASPD with alcoholism compared with alcoholics lacking ASPD and healthy controls. However, there were few ASPD subjects in this study relative to the other groups analyzed [15]. This result was replicated by another group reporting that the MAOA-L allele was more common in ASPD [16]. The association between the MAO-A VNTR genotype and DSM-IV [17] ASPD personality traits was tested in a community sample comprising 435 individuals. This investigation did not include fine-grained measures of early adverse events, as childhood maltreatment was assessed by asking subjects whether they had ever been disciplined too harshly by a caregiver. Raters were then instructed to probe individuals who endorsed the item and classify the presence or absence of abuse in a dichotomous fashion. Results indicated that among Caucasians without a history of childhood maltreatment, MAOA-L carriers had higher ASPD traits than subjects with MAOA-H. Caucasian MAOA-L carriers with no exposure to abuse additionally reported greater anger/hostility, lower agreeableness, and scored higher on a NEO-PI-R [18] index of prototypical ASPD than participants with the MAOA-H genotype [19]. In another study, the interactive effect of the MAO-A VNTR polymorphism and testosterone level sampled from cerebrospinal fluid was tested in a sample of 95 criminal alcoholics remanded to a forensic psychiatry unit and 45 healthy controls [20]. The authors reported that the combination of higher testosterone levels and the reduction-of-function MAO-A genotype predicted ASPD and greater lifetime aggression. The smaller sample size and suggestion that higher testosterone levels could be a consequence of ASPD were noted as limitations. Another longitudinal investigation examined the effect of childhood maltreatment on symptoms of ASPD and major depressive disorder (MDD) [21]. Lifetime symptoms of ASPD were measured over two waves of the study, albeit using an uncommon measure. Symptoms of depression were positively correlated with ASPD symptoms. Interestingly, the interaction between MAOA-L and childhood maltreatment predicted ASPD symptoms, whereas the combination of MAOA-H and childhood abuse was associated with depressive symptomatology. 
In a particularly robust study examining the genetic underpinnings of violent offending, a discovery cohort of both male and female Finnish prisoners $(n=794)$, many of whom had been diagnosed with ASPD, and members of a cohort of homicide offenders $(n=114)$, was categorized as either non-violent offenders, violent offenders who had committed at least one violent offense, or extreme violent offenders who were implicated in 10 or more offenses $[22 \bullet \cdot$. The authors reported a main effect of MAO-A genotype on violent outcomes, such that MAOA-L was linked to violent offending among members of the discovery cohort. The association between MAOA-L and violence was even stronger among extreme violent offenders derived from the same cohort. Notably, these relationships were unaffected by childhood maltreatment. Another investigation of a criminal population at high risk for violence reported that MAOA-L was associated with a psychopathy construct consisting of greater impulsivity and irresponsibility, even after controlling for other psychopathic symptoms [23].

With rare exception, candidate gene studies of MAO-A in ASPD have focused exclusively on males or mixed samples. The reason for this discrepancy relates to the fact that males are hemizygous for the MAO-A VNTR allele, whereas females are homozygous or heterozygous at the same locus. These differences make it difficult to compare genotypes between males and females. Furthermore, although X-inactivation occurs in females, some evidence suggests that the MAO-A gene may escape inactivation, thus potentially offsetting the effects of any low or non-functioning allele on the other X chromosome [24, 25]. One of the few studies to examine the relationship between MAO-AVNTR alleles and ASPD in females investigated a sample of Southwest American Indians [26]. The main objective of the experiment was to analyze the interaction between MAO-A genotype and childhood sexual abuse on development of alcoholism and ASPD. An important finding that emerged was that low-activity homozygotes who had suffered childhood sexual abuse had increased rates of alcoholism, ASPD, and more ASPD symptoms compared with subjects in possession of a high-activity allele. Retrospective reporting of childhood abuse and a relatively small sample size were main limitations of the investigation.

While the aforementioned studies report on associations between low-activity MAO-A genetic variants and ASPD, some studies have implicated MAOA-H in the pathogenesis of the illness. For example, a Finnish sample of 174 male violent offenders with alcohol use disorders, $36 \%$ of whom were diagnosed with ASPD, was genotyped for the MAO-A VNTR and assessed for the presence of childhood physical abuse. Offenders were followed in the community thereafter for a period of 8 years. Results indicated that childhood abuse predicted violent recidivism among those with the MAOA-H allele but not MAOA-L. A main study limitation was that childhood physical abuse was assessed retrospectively [27]. Another investigation by the same research group that analyzed an overlapping sample $(34 \%$ with
ASPD and 28\% with comorbid BPD and ASPD) reported that for every one point increase on the Psychopathy ChecklistRevised (PCL-R), a common tool for measuring psychopathic traits [28], risk for impulsive re-conviction increased by $6.8 \%$, but only in subjects with MAOA-H [29]. Both antisocial behavior and attitudes in this analysis predicted re-convictions to a greater degree in MAOA-H versus MAOA-L. Importantly, this study did not account for childhood maltreatment and thus was unable to ascertain whether gene-environment interactions may have moderated these relationships. Although this paper argued that MAOA-H was related to impulsive forms of violence, one study with a very small sample of ASPD violent offenders and healthy controls reported that the high-functioning MAO-A allele was more common in ASPD and that it predicted levels of proactive aggression [30]. On the other hand, possession of the high-activity allele may buffer the impact that association with delinquent peers has on development of adult criminal behavior [31]. It must be noted that some studies have failed to detect a relationship between MAO-A VNTR genetic variants and ASPD as well as gene-environment interactions contributing to antisocial outcomes [32]. In fact, the study with the largest sample size to date (3356 white and 960 black males) utilized the National Longitudinal Study of Adolescent Health to investigate the relationship between childhood maltreatment, conduct problems in adolescence, adult antisocial behavior, and the MAO-A VNTR [33]. Although main effects of the MAO-A gene on disposition toward violence and violent convictions were reported, these analyses did not survive correction for multiple testing.

\section{Other MAO-A Polymorphisms in ASPD}

Polymorphisms of the MAO-A gene in addition to the upstream VNTR have also been examined in ASPD. One recent study aimed to determine whether antisocial behavior could be predicted by the impact of the MAO-A gene on exposure to violence during childhood [34]. The research was carried out in adults who had been followed since childhood and who had been selected from a normative subsample of children or a subsample of youth with disruptive and antisocial behaviors. Five single nucleotide polymorphisms (SNPs) of the MAO-A gene were analyzed with main and interactive effects of these SNPs tested across the full range of exposure to violence. Another research question asked whether these polymorphisms increased risk of antisocial behavior among subjects exposed to higher levels of childhood violence. In general, among individuals exposed to violence, greater risk of antisocial behaviors was detected in males possessing low frequency MAO-A alleles compared with carriers of high frequency MAO-A alleles. Moreover, the nonlinear interactions identified between MAO-A SNPs and violence indicated that genetic modulation of risk factors only operated at a specific threshold of violence. Regarding the relationship between MAO-A and ASPD symptomatology, main effects 
were observed for five of the SNPs and ASPD symptoms after controlling for violence and maternal ASPD. Significant nonlinear associations were also present for ASPD symptoms and several of the MAO-A SNPs. The authors agreed that results could have been more informative had they also analyzed the MAO-A VNTR. A novel MAO-A VNTR (VNTR2) located approximately 1500 bps upstream of the previously defined VNTR has been identified [35]. It was subsequently determined that a $10 \mathrm{bp}$ allele at this location functions as a low-activity allele. In a novel study, researchers investigated whether a gene-environment interaction at this locus could predict ASPD. ASPD symptoms were diagnosed using a variety of well-validated instruments, while an index of maltreatment variables was used to measure childhood abuse. Results revealed that ASPD symptoms were not associated with the interaction of childhood abuse and VNTR2 or VNTR in males. However, among females, the combination of the low-activity VNTR2 allele and a history of child abuse was associated with symptoms of ASPD. Interestingly, in subjects with no low-activity alleles, childhood maltreatment was still significantly correlated with ASPD symptoms, although the correlation was weaker compared with participants with at least one low-activity allele. It was suggested by the authors that the VNTR2 may play a more influential role than VNTR with regard to transcriptional activity, but they also acknowledged that both loci were in close linkage disequilibrium and that marginal findings could have been due to chance.

\section{Epigenetic Investigations in ASPD}

Epigenetic studies have also advanced our understanding of how MAO-A regulation may affect manifestation of ASPD. An earlier study analyzed methylation patterns of two $\mathrm{CpG}$ islands flanking the MAO-A VNTR in an enriched sample of individuals at high risk for ASPD [36]. However, no relationship between ASPD symptom count and amount of methylation was found for either gender. Low power may have been an important contributor to these findings. A more recent investigation examined the methylation status of a $1.5 \mathrm{~kb}$ region of the MAO-A promoter in 86 incarcerated males meeting DSM-IV-TR [37] criteria for ASPD and 73 healthy controls [38•]. The investigators reported a general hyper-methylation of the MAO-A promoter region among the ASPD participants. Functional assessment of a region of interest within the MAO-A promoter demonstrated unambiguous promoter activity but downregulation of MAO-A activity. Finally, in a subsample of 80 ASPD participants, greater MAO-A promoter methylation was correlated with serotonin blood levels. These results, as suggested by the authors, could help explicate findings of decreased MAO-A activity and increased serotonin in ASPD, findings which have been previously reported in violent individuals [39]. However, the absence of any data on MAO-A enzyme activity precluded the ability to infer a causal relationship between MAO-A promoter hyper-methylation and abnormal MAO-A or serotonin activity. The relationship between peripheral and central methylation patterns also lacks clarity at this point in time.

\section{Neuroimaging MAO-A in ASPD}

A handful of neuroimaging studies, including techniques that can quantify in vivo levels of the MAO-A enzyme, have made important inroads to better understanding the neural substrate of ASPD and its symptoms. In one investigation, MAO-A genotypes were investigated in relation to the NEO Five-Factor Inventory Antisocial Index (FFI-AI), an instrument derived from the NEOFFI $[18,40]$ by the authors, for assessing ASPD or ASPD traits, and event-related potentials (ERPs) measured during automatic or controlled processing of emotional stimuli [41]. Results revealed that MAOA-L males exhibited altered anger-related ERPs in right medial frontal, left parietal, superior temporal, and superior occipital regions relative to MAOA-H carriers. Males possessing the MAOA-L allele tended to show alterations in ERPs that were positively correlated with FFI-AI scores. The authors postulated that the reduction-of-function MAO-A allele in males could enhance vulnerability to antisocial traits through modulation of neural systems responsible for evaluating threat-related information. The PET radiotracer $\left[{ }^{11} \mathrm{C}\right]$-harmine was used to measure MAO-A density in the orbitofrontal cortex (OFC) and ventral striatum (VS) in another study comprising 18 participants with ASPD and 18 healthy controls [42•]. All subjects were male. ASPD participants were diagnosed by a forensic psychiatrist using the Structured Clinical Interview for DSM-IV Axis II, Personality Disorders (SCID II) [43]. Study participants were nonsmoking and were not taking any psychotropic medication. Nine ASPD subjects met criteria for alcohol dependence (AD). The control group consisted of nine healthy individuals without a psychiatric history as determined by clinical assessment and nine subjects with $\mathrm{AD}$ to match against the ASPD subjects with AD. Results demonstrated that MAO-A density was significantly lower in ASPD in both the OFC and VS. Among the ASPD subsample, lower VS MAO-A density was associated with more impulsive decision-making on the Iowa Gambling Task [44], greater self-reported NEO-PI-R impulsivity, and elevated impulsivity assessed using the PCL-R. Lack of female participants was viewed as a study limitation. However, these findings were the first to demonstrate a link between pathological markers of MAO-A in preclinical models of aggression and central measures of MAO-A in ASPD. Another investigation that included a subsample of the previous study provided evidence in ASPD of a relationship between VS MAO-A density and functional connectivity of striatal regions assessed using functional magnetic resonance imaging [45]. For example, ventral striatal MAO-A density was shown to correlate with dorsolateral prefrontal cortex-superior ventral striatum functional connectivity. Conversely, ventral striatum MAO-A levels were negatively 
correlated with hippocampus-inferior ventral striatum functional connectivity. A main advantage of this study is that it was able to link in vivo levels of MAO-A, already shown to be abnormal in ASPD, to patterns of functional connectivity originating from the same region, thus reinforcing the notion that brain endophenotypes of ASPD can provide insight to understanding brain function. The main limitation of this study is that it did not include a control group.

\section{Borderline Personality Disorder}

BPD is a common condition that occurs in $10 \%$ of psychiatric outpatients and $15-20 \%$ of psychiatric inpatients [46]. Females with the disorder are over-represented in clinical settings, where they comprise approximately $70 \%$ of cases [47]. Several constellations of psychopathology, including affective, cognitive, impulsive, and interpersonal symptoms, characterize BPD. Compared with the amount of research that has been devoted to studying MAO-A in ASPD, fewer resources have been spent pursuing similar lines of investigation in BPD. Several reasons may explain this incongruity. The first suggested reason for the relative paucity of MAO-A research in BPD, as discussed earlier, is because MAO-A gene expression in females cannot be classified with absolute precision given discrepant findings as to whether $\mathrm{X}$-activation occurs at the MAO-A gene locus. Thus, it cannot be determined with certainty whether the level of MAO-A transcription is a product of one or two functioning MAO-A alleles [24, 25]. Since most studies of BPD involve clinical samples and females are more likely to occupy this segment of the population, researchers may be reluctant to carry out candidate gene studies of MAO-A in this group. A second hypothesized reason relates to the fact that classic symptoms of BPD, such as fear of abandonment or the presence of unstable relationships, have not been linked to MAO-A in the same way that antisocial behavior has shown a relationship with altered MAO-A in both clinical and non-clinical populations. What follows is the available genetic and neuroimaging research on MAO-A in BPD.

\section{MAO-A VNTR in BPD}

An early study examined the MAO-A VNTR genotype in BPD as part of a larger investigation that analyzed MAOA VNTR genetic variants in relation to the three personality disorder clusters [48]. A higher percentage of the low-activity MAO-A variant was found in all personality disorders except BPD. These analyses were likely underpowered as the BPD sample contained only 15 subjects. Another study that examined MAO-A polymorphic markers in BPD genotyped the MAO-A VNTR and an MAO-A SNP (rs6323) on exon 8 in 111 Caucasian males and females with BPD and 289 healthy controls of both sexes [49]. For the clinical group, psychosis, bipolar I disorder, dementia, current substance dependence, and brain injury were exclusionary. The results indicated a higher frequency of MAOA-H alleles among BPD subjects relative to controls and a lower frequency of the low-activity haplotype, which consisted of the lowactivity variant of the MAO-A VNTR and the T allele of rs6323. To help interpret their findings, the authors suggested that variability in the MAO-A gene could contribute to different types of psychopathology in BPD and other psychiatric disorders. The main limitation of this study was its small sample size. This same research group next genotyped 27 polymorphisms across seven serotonin genes in addition to the MAO-A VNTR in a sample of 113 Caucasians with BPD (it was unclear whether these subjects had participated in the previous investigation) and controls matched on age, sex, and ethnicity [50]. Results revealed a significant gene-gene interaction between MAO-A VNTR and three other serotonergic genotypes. These results reinforce the relevance of serotonin as an important neurochemical marker of BPD [51] and suggest that the interaction of MAO-A and serotonin polymorphisms may influence development of BPD. The most recent investigation to examine the relationship between MAO-A VNTR and BPD tested polymorphic distributions of the MAO-A VNTR genetic variant and four other genes (5-HTR2A, COMT, DAT, and 5-HTT) in a sample of 296 female patients dependent on heroin (63 with BPD) and 101 normal females [52]. A psychiatrist and psychologist diagnosed subjects using the SCID I [53]. Controls were screened by history, examination, and laboratory tests. A main finding was that opioid-dependent subjects with BPD had a higher frequency of the 4-repeat MAO-A VNTR allele than opioid-dependent participants without BPD. Interactive effects between the MAO-A VNTR and serotonergic genes approached significance in predicting comorbid BPD and opioid dependence. The small number of BPD subjects and lack of urine drug screening in the healthy control group were cited as study limitations. Interestingly, this same research group also examined the association and interactive effects of these same genes in samples of opioid-dependent males with ASPD, opioiddependent males without ASPD, and healthy controls. MAO-A was not implicated in any of the results [54].

\section{Neuroimaging in BPD}

One lone PET investigation has examined BPD and its symptom clusters in relation to MAO-A density [55 •*]. The principal aim of the study was to measure MAO-A density in the prefrontal cortex (PFC) and anterior cingulate cortex (ACC) of BPD. Secondary aims examined how MAO-A density was correlated 
with mood symptoms, suicidality, and neurocognitive impairment. $\left[{ }^{11} \mathrm{C}\right]$-Harmine PET was applied to four groups of 14 participants, all of whom were female: (1) BPD with severe symptoms, (2) BPD with moderate symptoms, (3) individuals with a major depressive episode (MDE) only, and (4) healthy controls. Concurrent MDE was designated an inclusion criterion for the BPD group to increase generalizability of the results, since MDD is present in $90 \%$ of female patients [56]. Therefore, a non-comorbid MDE group was included to control for the effect of MDE in the BPD subjects. BPD severity was based on the median split of the number of DSM-IV-TR symptoms present. Results revealed that MAO-A density in the PFC and ACC was significantly higher in the severe BPD group even after controlling for level of depressive symptoms. In the 28 BPD subjects, PFC and ACC density showed a positive relationship with depressive symptoms and suicidality, while hippocampal MAO-A density and verbal memory were negatively correlated. These findings suggested that central markers of MAO-A have relevance for elucidating the pathophysiology of $\mathrm{BPD}$, including suicidality and neurocognitive deficits. Limitations included the fact that a handful of BPD subjects were taking medication at the time of PET scanning. However, results did not change when these individuals were removed from the analyses. The absence of male participants also decreases generalizability to the entire BPD population.

\section{MAO Inhibitors as Treatments for BPD}

MAO inhibitors (MAOIs) were serendipitously discovered to be effective treatments for major depressive disorder (MDD) after depressed tuberculosis patients being treated with iproniazid, a derivative of isocarboxazid, experienced improved mood [57]. Given the relationship of MAO-A polymorphisms to depressed mood states [58,59], it is surprising that so little research has explored the relationship between MAO-A genetic variants and clinical response to these agents. An early study of depressed subjects concluded that MAO-A polymorphisms did not affect response to moclobemide, a reversible inhibitor of MAO-A. On the other hand, another study found that females with MDD in possession of two high-activity alleles exhibited a slower and less efficient antidepressant response [60]. The challenge of interpreting the results of the latter study is that patients were treated in a naturalistic setting that permitted the use of serotonin selective reuptake inhibitors, serotonin norepinephrine reuptake inhibitors, noradrenergic and specific serotonergic antidepressants, tricyclic antidepressants, benzodiazepines, mood stabilizers, and atypical antipsychotics, in addition to MAOIs. MAOIs have also been tested for their ability to treat symptom clusters of BPD. For example, one of the earliest medication trials conducted in BPD patients reported that tranylcypromine improved physician global ratings of depression and impulsivity [61]. A subsequent investigation that included a much larger sample size randomized 108 BPD subjects to phenelzine, haloperidol, or placebo [62]. Although improvements on state measures of depression were observed after 5 weeks of treatment with phenelzine, results did not differ from the placebo arm. Because ingestion of certain foods or medications can be lethal in patients taking MAOIs and intentional overdose can also lead to death, clinicians must proceed with caution when treating BPD patients with MAOIs.

\section{Conclusion}

MAO-A is one of the most intensely scrutinized molecular targets in the behavioral sciences [63], and abnormalities in MAOA structure and function have been implicated in ASPD and BPD. More research exists for ASPD than BPD, possibly because antisocial and conduct-disordered behaviors have been linked to alterations of MAO-A in non-clinical populations. For both ASPD and BPD, genetic association studies have contributed the most evidence by demonstrating that variation in the MAO-A gene occurs in both disorders. Although meta-analysis connects antisocial phenotypes to the low-activity MAO-A VNTR genetic variant [64], other studies report main effects of MAOA-H or describe gene-environment interactions influencing diagnosis and personality disorder symptomatology. Differences in the timing, type, and severity of childhood environmental stressors may help account for these discrepancies. Establishing genetic consortiums and pooled data analysis of ASPD and BPD cases would be particularly advantageous for future genetic research. Finally, conflicting results in studies of BPD and ASPD samples that have used identical neuroimaging techniques $[42 \bullet, 55 \bullet]$ or examined very similar populations $[52$, 54] further confirm the independence of ASPD and BPD as separate illnesses manifesting varied association with MAO-A.

\section{Compliance with Ethical Standards}

Conflict of Interest Ms. Sarah Vinette reports receiving a student research grant from the Mach-Gaensslen Foundation of Canada. Dr. Nathan Kolla declares that he has no conflicts of interest.

Human and Animal Rights and Informed Consent This article does not contain any studies with human or animal subjects performed by any of the authors.

\section{References}

Papers of particular interest, published recently, have been highlighted as:

- Of importance

•- Of major importance

1. Sabol SZ, Hu S, Hamer D. A functional polymorphism in the monoamine oxidase A gene promoter. Hum Genet. 1998;103(3):273-9. 
2. Huang YY, Cate SP, Battistuzzi C, Oquendo MA, Brent D, Mann JJ. An association between a functional polymorphism in the monoamine oxidase a gene promoter, impulsive traits and early abuse experiences. Neuropsychopharmacology. 2004;29(8):1498-505.

3. Deckert J, Catalano M, Syagailo YV, Bosi M, Okladnova O, Di Bella D, et al. Excess of high activity monoamine oxidase A gene promoter alleles in female patients with panic disorder. Hum Mol Genet. 1999;8(4):621-4.

4. Youdim MB, Edmondson D, Tipton KF. The therapeutic potential of monoamine oxidase inhibitors. Nat Rev Neurosci. 2006;7(4):295-309.

5. Alia-Klein N, Kriplani A, Pradhan K, Ma JY, Logan J, Williams B, et al. The MAO-A genotype does not modulate resting brain metabolism in adults. Psychiatry Res. 2008;164(1):73-6.

6. Fowler JS, Alia-Klein N, Kriplani A, Logan J, Williams B, Zhu W, et al. Evidence that brain MAO A activity does not correspond to MAO A genotype in healthy male subjects. Biol Psychiatry. 2007;62(4):355-8.

7. Cases O, Seif I, Grimsby J, Gaspar P, Chen K, Pournin S, et al. Aggressive behavior and altered amounts of brain serotonin and norepinephrine in mice lacking MAOA. Science. 1995;268(5218):1763-6.

8. Brunner HG, Nelen M, Breakefield XO, Ropers HH, van Oost BA. Abnormal behavior associated with a point mutation in the structural gene for monoamine oxidase A. Science. 1993;262(5133):578-80.

9. Brunner HG, Nelen MR, van Zandvoort P, Abeling NG, van Gennip AH, Wolters EC, et al. X-linked borderline mental retardation with prominent behavioral disturbance: phenotype, genetic localization, and evidence for disturbed monoamine metabolism. Am J Hum Genet. 1993;52(6):1032-9.

10. Palmer EE, Leffler M, Rogers C, Shaw M, Carroll R, Earl J, et al. New insights into Brunner syndrome and potential for targeted therapy. Clin Genet. 2016;89(1):120-7.

11. Caspi A, McClay J, Moffitt TE, Mill J, Martin J, Craig IW, et al. Role of genotype in the cycle of violence in maltreated children. Science. 2002;297(5582):851-4.

12. Swanson MC, Bland RC, Newman SC. Epidemiology of psychiatric disorders in Edmonton. Antisocial personality disorders. Acta Psychiatr Scand Suppl. 1994;376:63-70.

13. Fazel S, Danesh J. Serious mental disorder in 23000 prisoners: a systematic review of 62 surveys. Lancet. 2002;359(9306):545-50.

14. Coid J, Yang M, Roberts A, Ullrich S, Moran P, Bebbington P, et al. Violence and psychiatric morbidity in the national household population of Britain: public health implications. Br J Psychiatry. 2006;189:12-9.

15. Samochowiec J, Lesch KP, Rottmann M, Smolka M, Syagailo YV, Okladnova $\mathrm{O}$, et al. Association of a regulatory polymorphism in the promoter region of the monoamine oxidase A gene with antisocial alcoholism. Psychiatry Res. 1999;86(1):67-72.

16. Schmidt LG, Sander T, Kuhn S, Smolka M, Rommelspacher H, Samochowiec J, et al. Different allele distribution of a regulatory MAOA gene promoter polymorphism in antisocial and anxiousdepressive alcoholics. J Neural Transm. 2000;107(6):681-9.

17. Author. Diagnostic and statistical manual of mental disorders, 4th edition. Washington, D.C.: American Psychiatric Association; 1994.

18. Costa PT, McCrae RR. Revised NEO personality inventory (NEOPI-R) and NEO five-factor inventory (NEO-FFI). Psychological Assessment Resources: Odessa, FL; 1992.

19. Reti IM, Xu JZ, Yanofski J, McKibben J, Uhart M, Cheng YJ, et al. Monoamine oxidase A regulates antisocial personality in whites with no history of physical abuse. Compr Psychiatry. 2011;52(2):188-94.

20. Sjoberg RL, Ducci F, Barr CS, Newman TK, Dell'osso L, Virkkunen M, et al. A non-additive interaction of a functional MAO-A VNTR and testosterone predicts antisocial behavior. Neuropsychopharmacology. 2008;33(2):425-30.

21. Beach SR, Brody GH, Gunter TD, Packer H, Wernett P, Philibert RA. Child maltreatment moderates the association of MAOA with symptoms of depression and antisocial personality disorder. J Fam Psychol. 2010;24(1):12-20.

22.• Tiihonen J, Rautiainen MR, Ollila HM, Repo-Tiihonen E, Virkkunen M, Palotie A, et al. Genetic background of extreme violent behavior. Mol Psychiatry. 2015;20(6):786-92. This study demonstrated that the low activity MAO-A genetic variant was associated with violent offenders and that the relationship was even stronger between the same genotype and seriously violent offenders

23. Sadeh N, Javdani S, Verona E. Analysis of monoaminergic genes, childhood abuse, and dimensions of psychopathy. J Abnorm Psychol. 2013;122(1):167-79.

24. Benjamin D, Van Bakel I, Craig IW. A novel expression based approach for assessing the inactivation status of human X-linked genes. Eur J Hum Genet. 2000;8(2):103-8.

25. Carrel L, Willard HF. X-inactivation profile reveals extensive variability in X-linked gene expression in females. Nature. 2005;434(7031):400-4.

26. Ducci F, Enoch MA, Hodgkinson C, Xu K, Catena M, Robin RW, et al. Interaction between a functional MAOA locus and childhood sexual abuse predicts alcoholism and antisocial personality disorder in adult women. Mol Psychiatry. 2008;13(3):334-47.

27. Tikkanen R, Ducci F, Goldman D, Holi M, Lindberg N, Tiihonen J, et al. MAOA alters the effects of heavy drinking and childhood physical abuse on risk for severe impulsive acts of violence among alcoholic violent offenders. Alcohol Clin Exp Res. 2010;34(5):853-60.

28. Hare R. Hare psychopathy checklist-revised. 2nd ed. Toronto: Multi-Health Systems;2003.

29. Tikkanen R, Auvinen-Lintunen L, Ducci F, Sjoberg RL, Goldman D, Tiihonen J, et al. Psychopathy, PCL-R, and MAOA genotype as predictors of violent reconvictions. Psychiatry Res. 2011;185(3):382-6.

30. Kolla NJ, Attard S, Craig G, Blackwood N, Hodgins S. Monoamine oxidase A alleles in violent offenders with antisocial personality disorder: high activity associated with proactive aggression. Crim Behav Ment Health. 2014;24(5):368-72.

31. Lu YF, Menard S. The interplay of MAOA and peer influences in predicting adult criminal behavior. Psychiatric Q. 2016:1-14

32. Lu RB, Lin WW, Lee JF, Ko HC, Shih JC. Neither antisocial personality disorder nor antisocial alcoholism is associated with the MAO-A gene in Han Chinese males. Alcohol Clin Exp Res. 2003;27(6):889-93.

33. Haberstick BC, Lessem JM, Hewitt JK, Smolen A, Hopfer CJ, Halpern CT, et al. MAOA genotype, childhood maltreatment, and their interaction in the etiology of adult antisocial behaviors. Biol Psychiatry. 2014;75(1):25-30.

34. Ouellet-Morin I, Cote SM, Vitaro F, Hebert M, Carbonneau R, Lacourse E, et al. Effects of the MAOA gene and levels of exposure to violence on antisocial outcomes. Br J Psychiatry. 2016;208(1): $42-8$.

35. Philibert RA, Wernett P, Plume J, Packer H, Brody GH, Beach SR. Gene environment interactions with a novel variable monoamine oxidase A transcriptional enhancer are associated with antisocial personality disorder. Biol Psychol. 2011;87(3):366-71.

36. Philibert RA, Gunter TD, Beach SR, Brody GH, Madan A. MAOA methylation is associated with nicotine and alcohol dependence in women. Am J Med Genet B Neuropsychiatr Genet. 2008;147B(5): $565-70$.

37. Author. Diagnostic and statistical manual of mental disorders 4th edition, text revision Washington, D.C.: American Psychiatric Association; 2000.

38. Checknita D, Maussion G, Labonte B, Comai S, Tremblay RE, Vitaro F, et al. Monoamine oxidase A gene promoter methylation and transcriptional downregulation in an offender population with antisocial personality disorder. Br J Psychiatry. 2015;206(3):21622. MAO-A promoter methylation was shown to be increased in ASPD, while downregulation of MAO-A gene expression was also observed 
39. Moffitt TE, Brammer GL, Caspi A, Fawcett JP, Raleigh M, Yuwiler A, et al. Whole blood serotonin relates to violence in an epidemiological study. Biol Psychiatry. 1998;43(6):446-57.

40. Costa PT, McCrae RR. The NEO PI/NEO-FFI manual supplement. Odessa, FL: Psychological Assessment Sources; 1989.

41. Williams LM, Gatt JM, Kuan SA, Dobson-Stone C, Palmer DM, Paul $\mathrm{RH}$, et al. A polymorphism of the MAOA gene is associated with emotional brain markers and personality traits on an antisocial index. Neuropsychopharmacology. 2009;34(7):1797-809.

42. Kolla NJ, Matthews B, Wilson AA, Houle S, Bagby RM, Links P, et al. Lower monoamine oxidase-A total distribution volume in impulsive and violent male offenders with antisocial personality disorder and high psychopathic traits: an [(11)C] harmine positron emission tomography study. Neuropsychopharmacology. 2015;40(11):2596-603. This study was the first to demonstrate that MAO-A brain density is lower in ASPD. These results accord well with preclinical work of MAO-A function

43. First MB, Gibbon M, Spitzer RL, Williams JBW, Benjamin LS. Structured clinical interview for DSM-IV Axis II personality disorders, (SCID-II). Washington, D.C.: American Psychiatric Press, Inc.; 1997.

44. Bechara A, Damasio AR, Damasio H, Anderson SW. Insensitivity to future consequences following damage to human prefrontal cortex. Cognition. 1994;50(1-3):7-15.

45. Kolla NJ, Dunlop K, Downar J, Links P, Bagby RM, Wilson AA, et al. Association of ventral striatum monoamine oxidase-A binding and functional connectivity in antisocial personality disorder with high impulsivity: a positron emission tomography and functional magnetic resonance imaging study. Eur Neuropsychopharmacol. 2016;26(4):777-86.

46. Torgersen S, Kringlen E, Cramer V. The prevalence of personality disorders in a community sample. Arch Gen Psychiatry. 2001;58(6):590-6.

47. Korzekwa MI, Dell PF, Links PS, Thabane L, Webb SP. Estimating the prevalence of borderline personality disorder in psychiatric outpatients using a two-phase procedure. Compr Psychiatry. 2008;49(4):380-6.

48. Jacob CP, Muller J, Schmidt M, Hohenberger K, Gutknecht L, Reif A, et al. Cluster $\mathrm{B}$ personality disorders are associated with allelic variation of monoamine oxidase A activity. Neuropsychopharmacology. 2005;30(9):1711-8.

49. Ni X, Sicard T, Bulgin N, Bismil R, Chan K, McMain S, et al. Monoamine oxidase a gene is associated with borderline personality disorder. Psychiatr Genet. 2007;17(3):153-7.

50. Ni X, Chan D, Chan K, McMain S, Kennedy JL. Serotonin genes and gene-gene interactions in borderline personality disorder in a matched case-control study. Prog Neuropsychopharmacol Biol Psychiatry. 2009;33(1):128-33.

51. Soloff PH, Meltzer CC, Greer PJ, Constantine D, Kelly TM. A fenfluramine-activated FDG-PET study of borderline personality disorder. Biol Psychiatry. 2000;47(6):540-7.

52. Yang M, Mamy J, Wang Q, Liao YH, Seewoobudul V, Xiao SY, et al. The association of 5-HTR2A-1438A/G, COMTVal158Met,
MAOA-LPR, DATVNTR and 5-HTTVNTR gene polymorphisms and borderline personality disorder in female heroin-dependent Chinese subjects. Prog Neuropsychopharmacol Biol Psychiatry. 2014;50:74-82.

53. First MB, Spitzer RL, Gibbon M, Williams JBW. Structured clinical interview for DSM-IV-TR Axis I disorders, research version, patient edition (SCID-I/P), version 2. Biometrics Research, New York State Psychiatric Institute: New York, NY; 2002.

54. Yang M, Kavi V, Wang W, Wu Z, Hao W. The association of 5HTR2A-1438A/G, COMTVal158Met, MAOA-LPR, DATVNTR and 5-HTTVNTR gene polymorphisms and antisocial personality disorder in male heroin-dependent Chinese subjects. Prog Neuropsychopharmacol Biol Psychiatry. 2012;36(2):282-9.

55.• Kolla NJ, Chiuccariello L, Wilson AA, Houle S, Links P, Bagby $\mathrm{RM}$, et al. Elevated monoamine oxidase-A distribution volume in borderline personality disorder is associated with severity across mood symptoms, suicidality, and cognition. Biol Psychiatry. 2016;79(2):117-26. This investigation is the one neuroimaging study of MAO-A in BPD. Elevated MAO-A brain density was observed in several brain regions previously shown to be abnormal in BPD. MAO-A total distribution volume also correlated with mood symptoms and suicidality

56. Zanarini MC, Frankenburg FR, Dubo ED, Sickel AE, Trikha A, Levin A, et al. Axis I comorbidity of borderline personality disorder. Am J Psychiatry. 1998;155:1733-9.

57. Lopez-Munoz F, Alamo C. Monoaminergic neurotransmission: the history of the discovery of antidepressants from 1950s until today. Curr Pharm Des. 2009;15(14):1563-86.

58. Zhang J, Chen Y, Zhang K, Yang H, Sun Y, Fang Y, et al. A cisphase interaction study of genetic variants within the MAOA gene in major depressive disorder. Biol Psychiatry. 2010;68(9):795-800.

59. Doornbos B, Dijck-Brouwer DA, Kema IP, Tanke MA, van Goor SA, Muskiet FA, et al. The development of peripartum depressive symptoms is associated with gene polymorphisms of MAOA, 5HTT and COMT. Prog Neuropsychopharmacol Biol Psychiatry. 2009;33(7):1250-4.

60. Domschke K, Hohoff C, Mortensen LS, Roehrs T, Deckert J, Arolt $\mathrm{V}$, et al. Monoamine oxidase A variant influences antidepressant treatment response in female patients with major depression. Prog Neuropsychopharmacol Biol Psychiatry. 2008;32(1):224-8.

61. Cowdry RW, Gardner DL. Pharmacotherapy of borderline personality disorder. Alprazolam, carbamazepine, trifluoperazine, and tranylcypromine. Arch Gen Psychiatry. 1988;45(2):111-9.

62. Soloff PH, Cornelius J, George A, Nathan S, Perel JM, Ulrich RF. Efficacy of phenelzine and haloperidol in borderline personality disorder. Arch Gen Psychiatry. 1993;50(5):377-85.

63. Craig IW. The importance of stress and genetic variation in human aggression. Bioessays. 2007;29(3):227-36.

64. Ficks CA, Waldman ID. Candidate genes for aggression and antisocial behavior: a meta-analysis of association studies of the 5HTTLPR and MAOA-uVNTR. Behav Genet. 2014;44(5):427-44. 